\title{
Compression Changes in Electrical Resistance and Critical Temperature of $\mathrm{Nb}_{1-x} \mathrm{Se}_{2} \mathrm{Sn}_{x}$ Single Crystals
}

\author{
M.A. Obolenskiy ${ }^{a}$, D.D. Balla, A.A. Zavgorodniy ${ }^{a}$, R.V. VovK ${ }^{a}, *$ Z.F. NAzYrov ${ }^{a}$, \\ I.L. GOUlAtis ${ }^{a}$, M. JANUSZCZYK ${ }^{b}$ AND J.N. LATOSIŃSKA ${ }^{b}$ \\ ${ }^{a}$ V.N. Karazin Kharkiv National University, 4 Svoboda Sq., 61077 Kharkov, Ukraine \\ ${ }^{b}$ Adam Mickiewicz University, Umultowska 85, 61-614 Poznań, Poland
}

(Received May 20, 2011; in final form March 9, 2012)

\begin{abstract}
In this work we investigate the influence of hydrostatic pressure (up to $12 \mathrm{kbar}$ ) on the critical parameters of $\mathrm{Nb}_{1-x} \mathrm{Se}_{2} \mathrm{Sn}_{x}(x=0.1$ and $x=0.15)$ single crystals. It is shown that a change in the density of states at the Fermi level brings the main contribution to the increase in $T_{\mathrm{c}}$ under pressure. Under the effect of hydrostatic pressure, the phonon spectrum is shifted to higher frequencies (narrowed). The estimated Debye temperature is consistent with those obtained from the heat capacity measurements in $\mathrm{NbSe}_{2}$ single crystals. It is shown that upon increasing hydrostatic pressure the mean free path increases and $\mathrm{Nb}_{1-x} \mathrm{Se}_{2} \mathrm{Sn}_{x}$ should be considered as the pure limit in the Ginzburg-Landau theory.
\end{abstract}

PACS: 74.25.Fy, 74.62.Fj

\section{Introduction}

The discovery of high temperature superconductivity (HTSC) in 1986 in non-stoichiometric oxides gave a new rush of interest in the study of layered transition metal dichalcogenides (TMDs) [1], which have a strong anisotropy of their physical characteristics. Identification of similarities and differences in physical characteristics between the low and high temperature superconductors, coupled with determination of the crystal structure and mechanism of superconductivity [2] is an important task of solid state physics.

Anisotropy in TMDs, in particular in $\mathrm{NbSe}_{2}$ compounds, having the highest critical temperature $\left(T_{\mathrm{c}}\right)$ among TMDs, could be significantly enhanced [3] by introducing foreign atoms or molecules in the interlayer space (intercalation process). The intercalation ability of these compounds is related to the expected implementation of high-temperature superconductivity in the "sandwich" type structures described by the excitonic mechanism proposed by Ginzburg [3].

In addition, TMDs are characterized by the presence of a structural phase transition induced by the charge density wave (CDW). The variation in the CDW transition temperature $T_{\mathrm{c}}$ is the subject of intense theoretical and experimental studies [2, 3]. The experimental methods applied to identify the structural parameters of the superconductors which influence their superconducting characteristics and to allow determination of empirical ways to increase their critical parameters, such as the use of high hydrostatic pressure, are of special significance [4].

* corresponding author; e-mail:

Ruslan.V.Vovk@univer.kharkov.ua
In this work, we investigate the influence of high hydrostatic pressure up to $12 \mathrm{kbar}$ on the electric transport properties in the normal and superconducting compounds $\mathrm{Nb}_{1-x} \mathrm{Se}_{2} \mathrm{Sn}_{x}$ obtained by doping with tin at various concentrations as an impurity.

\section{Experimental techniques}

The single crystals were grown using the technology explained in detail in [5]. The hydrostatic pressure was produced in an autonomous chamber of the piston-cylinder type with a fixed clamp. To transfer the pressure we used a mixture of oil-kerosene in the ratio of $1: 1$.

The pressure was measured using a manganese manometer. The pressure fall in the chamber when it was cooled to the boiling point of liquid nitrogen was estimated on the basis of the pressure dependence of resistivity. The temperature measurements were performed using a copper-constantan thermocouple at $T>50 \mathrm{~K}$, with an error of $0.05 \mathrm{~K}$ and in the interval $4.2<T<50 \mathrm{~K}$ with a coal thermistor type TSU-2, with an error of $0.01 \mathrm{~K}$. The resistivity measurements were performed with the standard four-contact scheme.

\section{Results and discussion}

The influence of pressure on resistivity and the critical temperatures corresponding to the superconducting transition for different concentrations of tin in $\mathrm{Nb}_{1-x} \mathrm{Se}_{2} \mathrm{Sn}_{x}$ are shown in Fig. 1.

For all the investigated impurity concentrations of tin the typical peculiarities of the $R(T)$ behaviour correlated with the emergence of CDW transition are absent. This suggests that the CDW transition in these samples either is totally absent or it is significantly suppressed, and thus its influence should not have an effect on the temperature 


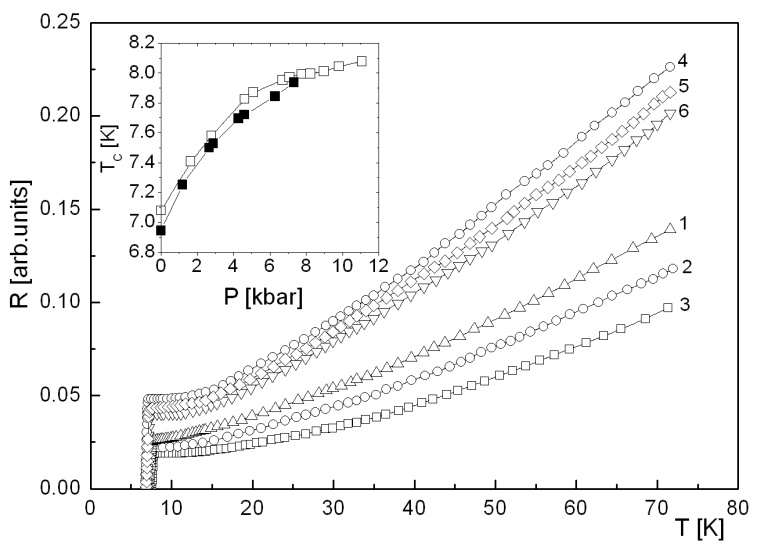

Fig. 1. Temperature dependences $R(T)$ for $\mathrm{Nb}_{1-x} \mathrm{Se}_{2} \mathrm{Sn}_{x}$ single crystals at different pressures and concentrations: for $x=0.1$ and $P=0(1), 4.5(2)$, 8.5 (3) kbar, for $x=0.15$ and $P=0$ (4), 5.7 (5), 12.8 (6) kbar. The inset shows the pressure dependence of the critical temperature for $x=0.1$ (open symbols) and $x=0.15$ (solid symbols).

of the superconducting transition. As shown in the inset to Fig. 1 at $P=0$ the increase in tin concentration leads to a decrease in $T_{\mathrm{c}}$.

The application of hydrostatic pressure leads to a sharp increase in $T_{\mathrm{c}}$ for the sample with $x=0.1$, at the rate $\mathrm{d} T_{\mathrm{c}} / \mathrm{d} P=0.2 \mathrm{~K} / \mathrm{kbar}$ under $P<4$ kbar and $\mathrm{d} T_{\mathrm{c}} / \mathrm{d} P=$ $0.04 \mathrm{~K} / \mathrm{kbar}$ in the interval $4<P<12 \mathrm{kbar}$. When the concentration of tin increases up to $x=0.15$, the bending point on the curve $T_{\mathrm{c}}(P)$ shifts to a lower pressure. The value of the slope $\mathrm{d} T_{\mathrm{c}} / \mathrm{d} P$ becomes $0.22 \mathrm{~K} / \mathrm{kbar}$ in the interval $0<P<3 \mathrm{kbar}$ and $0.08 \mathrm{~K} / \mathrm{kbar}$ at $P>3 \mathrm{kbar}$.

For the quantitative analysis of the $T_{\mathrm{c}}(P)$ dependence, we used the well-known McMillan formula [6]:

$$
T_{\mathrm{c}}=\frac{\theta_{\mathrm{D}}}{1.45} \exp \left(-\frac{1.04(1+\lambda)}{\lambda-\mu^{*}(1+0.62 \lambda)}\right),
$$

where $\theta_{\mathrm{D}}$ is the Debye temperature, $\mu^{*}$ - the screened Coulomb pseudopotential, characteristic for the electron repulsion, $\lambda$ - the electron-phonon interaction constant. The value of $\lambda$ depends on the parameters derived from the electronic and phonon spectra of metals

$$
\lambda=\frac{N\left(E_{\mathrm{F}}\right)\left\langle I^{2}\left(\boldsymbol{k}-\boldsymbol{k}^{\prime}\right)\right\rangle}{M \theta_{\mathrm{D}}^{2}},
$$

where $N\left(E_{\mathrm{F}}\right)$ is the density of states at the Fermi level, $\langle I\rangle$ is the averaged matrix element of electron-phonon interaction over the Fermi surface, $M$ is the ion mass.

As results from Eq. (2), with the introduction of the heavy impurity of tin, $T_{\mathrm{c}}$ decreases because $\theta_{\mathrm{D}} \sim \frac{1}{\sqrt{M}}$. Using the values $\lambda=0.81$ and $\mu^{*}=0.1$ [5] we can estimate the Debye temperature for $\mathrm{Nb}_{1-x} \mathrm{Se}_{2} \mathrm{Sn}_{x}$ at $P=0$, which is 177.8 and $175.5 \mathrm{~K}$ for the $x=0.1$ and $x=0.15$, respectively. These values $\theta_{\mathrm{D}}$ are in good agreement with the data obtained from the heat (thermal) capacity measurements [2]. According to Eq. (1), the Debye tempera- ture increases with increasing pressure as $T_{\mathrm{c}}$ increases.

According to (1), the $T_{\mathrm{c}}(P)$ dependence is determined by the quantities $\theta_{\mathrm{D}}, N\left(E_{\mathrm{F}}\right), \lambda, \mu^{*}$. It is clearly seen from the formula for $T_{\mathrm{c}}$ derived from the BCS theory

$$
\begin{aligned}
& T_{\mathrm{c}} \approx \theta_{\mathrm{D}} \exp \left(-\frac{1}{N\left(E_{\mathrm{F}}\right) V^{*}}\right), \\
& \frac{\partial T_{\mathrm{c}}}{\partial P}=\frac{\partial \theta_{\mathrm{D}}}{\partial P} \exp \left(-\frac{1}{N V^{*}}\right) \\
& \quad+\theta_{\mathrm{D}} \exp \left(-\frac{1}{N V^{*}}\right)\left(-\frac{1}{N V^{*}}\right)^{2} \frac{\partial\left(N V^{*}\right)}{\partial P},
\end{aligned}
$$

where $V^{*}$ is the matrix element of the scattering interaction.

The approximated $\theta_{\mathrm{D}}(P)$ dependence can be obtained using the Grüneisen formula describing the volume expansion coefficient [7]:

$$
\frac{1}{\theta_{\mathrm{D}}} \frac{\partial \theta_{\mathrm{D}}}{\partial P}=\frac{\alpha V}{C_{V}}
$$

where $\alpha$ is the thermal expansion coefficient, $V$ is volume and $C_{V}$ is the specific heat capacity at a constant volume.

As follows from Eq. (4), the Debye temperature should rise under hydrostatic pressure. The growth of $\theta_{\mathrm{D}}$ under pressure satisfactory explains the pressure-induced decrease in electrical resistance, and should lead to an increase in $T_{\mathrm{c}}$ (see Eq. (1)). However, the determination of $\theta_{\mathrm{D}}$ with the use of Eq. (1) is tentative and for $\mathrm{Nb}_{1-x} \mathrm{Se}_{2} \mathrm{Sn}_{x}$ gives values of $T_{\mathrm{c}}$ by an order of magnitude lower than the observed one. Thus, the change in $\theta_{\mathrm{D}}$, apparently, is not the main factor that determines the $T_{\mathrm{c}}(P)$ dependence.

We estimated the change in the density of states at the Fermi level under hydrostatic pressure, on the basis of the McMillan formula

$$
T_{\mathrm{c}}=\frac{\langle\omega\rangle}{1.22} \exp \left(-\frac{1.04(1+\lambda)}{\lambda-\mu^{*}(1+0.62 \lambda)}\right),
$$

where $\langle\omega\rangle$ is the average frequency of the phonon spectrum [8], assuming that the Coulomb potential $\mu^{*}$ weakly depends on pressure. Subsequently, the $T_{\mathrm{c}}(P)$ dependence is determined by the average frequency of the phonon spectrum and the electron-phonon constant $\lambda$.

If we write $\lambda$ as the ratio of the electron and lattice factors

$$
\lambda=\frac{\alpha}{M\left\langle\omega^{2}\right\rangle}, \quad \alpha=N\left(E_{\mathrm{F}}\right)\langle I\rangle^{2},
$$

and differentiate $T_{\mathrm{c}}$ with respect to the pressure, we obtain the following equation:

$$
\begin{aligned}
& \frac{\mathrm{d} \ln T_{\mathrm{c}}}{\mathrm{d} P}=\frac{1}{B}\left\{\gamma_{\mathrm{g}}+\frac{1.04 \lambda}{\lambda-\mu^{*}(1+0.62 \lambda)}\right. \\
& \left.-\frac{1.04 \lambda(1+\lambda)(1-0.62 \lambda)}{\left[1-\mu^{*}(1+0.62 \lambda)^{2}\right]^{2}} 2 \gamma+\frac{\mathrm{d} \ln \alpha}{\mathrm{d} \ln V}\right\},
\end{aligned}
$$

where $B=-\frac{V \mathrm{~d} P}{\mathrm{~d} V}$ is the volume modulus and $\gamma_{\mathrm{g}}$ is the 
Grüneisen effective constant

$$
\gamma_{\mathrm{g}}=-\frac{\mathrm{d} \ln \left\langle\omega^{2}\right\rangle^{1 / 2}}{\mathrm{~d} \ln V} .
$$

To estimate the last term in Eq. (7), we use the experimental data on $\mathrm{d} T_{\mathrm{c}} / \mathrm{d} P$ and since $\gamma_{\mathrm{g}}$ cannot be directly obtained from the experiment, we use the $\gamma_{\mathrm{g}}$ values taken from [8]. The results are listed in Table.

TABLE

Pressure derivatives estimated for the $\mathrm{Nb}_{1-x} \mathrm{Se}_{2} \mathrm{Sn}_{x}$ compounds.

\begin{tabular}{c|c|c|c|c}
\hline \hline \multirow{3}{*}{ Compound } & \multicolumn{2}{|c|}{$0<P<3 \mathrm{kbar}$} & \multicolumn{2}{|c}{$3<P<12 \mathrm{kbar}$} \\
\cline { 2 - 5 } & $\begin{array}{c}\mathrm{d} T / \mathrm{d} P \\
{[\mathrm{~K} / \mathrm{kbar}]}\end{array}$ & $\begin{array}{c}\mathrm{d} \ln \alpha / \mathrm{d} V \\
{\left[\mathrm{~m}^{-3}\right]}\end{array}$ & $\begin{array}{c}\mathrm{d} T / \mathrm{d} P \\
{[\mathrm{~K} / \mathrm{kbar}]}\end{array}$ & $\begin{array}{c}\mathrm{d} \ln \alpha / \mathrm{d} V \\
{\left[\mathrm{~m}^{-3}\right]}\end{array}$ \\
\hline $\mathrm{Nb}_{0.9} \mathrm{Se}_{2} \mathrm{Sn}_{0.1}$ & 0.2 & 19 & 0.04 & 3.7 \\
$\mathrm{Nb}_{0.85} \mathrm{Se}_{2} \mathrm{Sn}_{0.15}$ & 0.22 & 20 & 0.08 & 7
\end{tabular}

Table shows that the relative change in the density of states at the Fermi level is especially profound at pressures up to $3 \mathrm{kbar}$, which apparently predetermines the rapid increase in $T_{\mathrm{c}}$ with the application of hydrostatic pressure up to $3 \mathrm{kbar}$. In the pressure interval $3<P<12 \mathrm{kbar}$, the relative value of changes in the density of states decreases and slows the growth of $T_{\mathrm{c}}$. Consequently, the increase in $T_{\mathrm{c}}$ is mainly determined by changes in the density of states at the Fermi level and the change in the phonon spectrum plays a minor role. For this range of $T_{\mathrm{c}}$, the phonon spectrum becomes "harder". Using the Lifshitz formula for conductivity [9] we can find the mean free path and estimate its changes under pressure

$$
l \approx 1.27 \times 10^{4}\left[\rho_{\mathrm{res}} n^{2 / 3}\left(S / S_{\mathrm{F}}\right)\right]^{-1},
$$

where $l$ is the mean free path, $\rho_{\text {res }}$ is the residual resistance, $n$ is the carriers density, $S / S_{\mathrm{F}}$ is the ratio of the real Fermi surface to the Fermi surface of the free-electron gas. The calculations are performed under the assumption that the introduction of tin impurities does not significantly change the carriers' concentration and the area of the Fermi surface [9]. The change in residual resistivity under hydrostatic pressure is a result of the changes in the mean free path when the value $\rho_{\text {res }} n^{2 / 3}\left(S / S_{\mathrm{F}}\right)$ remains unchanged. The value $\rho_{\text {res }} n^{2 / 3}\left(S / S_{\mathrm{F}}\right)$ equal to $(4-6) \times 10^{14}$ is taken from [10].

The $l(P)$ dependence obtained using this approach is shown in Fig. 2. Comparing the mean free path to the coherence length, $\xi$, from the Ginzburg-Landau theory [11], we can see that $l>\xi$ and consequently the $\mathrm{Nb}_{1-x} \mathrm{Se}_{2} \mathrm{Sn}_{x}$ compound can be considered in the "pure" limit. Moreover, its "purity" increases with the rise of the hydrostatic pressure.

\section{Conclusions}

The peculiarities in the $R(T)$ dependences in $\mathrm{Nb}_{1-x} \mathrm{Se}_{2} \mathrm{Sn}_{x}$ compounds are due to the appearance of the CDW transition, whereas such behaviour does not occur in all samples with tin at different concentrations

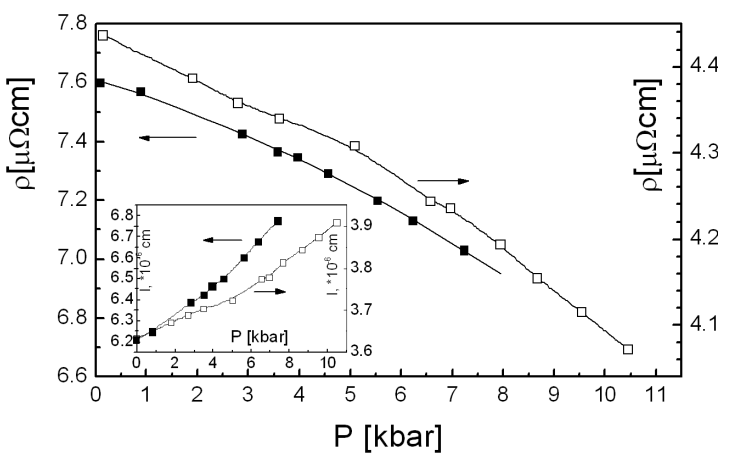

Fig. 2. Pressure dependence of residual resistivity for $\mathrm{Nb}_{0.85} \mathrm{Se}_{2} \mathrm{Sn}_{0.15}$ and $\mathrm{Nb}_{0.9} \mathrm{Se}_{2} \mathrm{Sn}_{0.10}$ single crystals, light and dark symbols, respectively. The inset shows the pressure dependence of the mean free path. The axis legend in the inset corresponds to the axis notation in the figure.

as an impurity. This suggests that the CDW-transition in the samples studied either is completely absent or it is significantly suppressed, and thus its influence on the superconducting transition temperature should not be reflected. The main contribution to the increase in $T_{\mathrm{c}}$ under the influence of high hydrostatic pressure on the $\mathrm{Nb}_{1-x} \mathrm{Se}_{2} \mathrm{Sn}_{x}$ results from the change in the density of states at the Fermi level. In this case, the phonon spectrum is shifted to higher frequencies (is narrower). The carriers' mean free path increases upon increasing hydrostatic pressure and $\mathrm{Nb}_{1-x} \mathrm{Se}_{2} \mathrm{Sn}_{x}$ should be considered in the pure limit in the Ginzburg-Landau theory.

\section{References}

[1] M.D. Johannes, I.I. Mazin, C.A. Howells, Phys Rev. B 73, 205102 (2006).

[2] V. Eremenko, V. Sirenko, V. Ibulaev, J. Bartolome, A. Arauzo, G. Remenyi, Physica C 469, 259 (2009).

[3] Problems of High- $T_{\mathrm{c}}$ Superconductivity, Eds. W.L. Ginzburg, D.A. Kirzhits, Nauka, Moskva 1977, p. 243 (in Russian).

[4] H. Suderow, V.G. Tissen, J.P. Brison, J.L. Martinez, S. Vieira, Phys. Rev. Lett. 95, 117006 (2005).

[5] Kh.B. Tchashka, E.I. Byletskiy, M.A. Obolenskiy, Fiz. Nisk. Temp. 17, 833 (1991) (in Russian).

[6] W.L. McMillan, Phys. Rev. 167, 331 (1968).

[7] Yu.N. Ryabinin, K.P. Rodyonov, E.S. Alekseyev, J. Theor. Phys. 34, 1913 (1964) (in Russian).

[8] C.W. Vonsovskiy, Yu.A. Izyumov, E. Kurmaev, Superconductivity of Transition Metals, their Alloys and Compounds, Nauka, Moskva 1977, p. 383 (in Russian).

[9] L.D. Landau, E.M. Lifshitz, Statistical Physics, part 1, Nauka, Moskva 1976, p. 226 (in Russian).

[10] D.E. Prober, R.E. Sehwal, H.R. Beasley, Phys. Rev. B 21, 2717 (1980).

[11] W.W. Schmidt, Introduction to Physics of Superconductors, MTNMO, Nauka, Moskva 2000, p. 322 (in Russian). 